\title{
Gravity Tests with Pulsars
}

\author{
Michael Kramer ${ }^{1,2}$ \\ ${ }^{1}$ MPI für Radioastronomie, \\ Auf dem Hügel 69, 53121 Bonn, Germany \\ email: mkramer@mpifr-bonn.mpg. de \\ ${ }^{2}$ Jodrell Bank Centre for Astrophysics, University of Manchester, \\ Manchester M13 9PL, UK
}

\begin{abstract}
Fifty years of pulsars also mean fifty years of using them as tools to probe other phenomena and physics. One prominent example is the usage of pulsars to test theories of gravity. Probing the quasi-stationary strong-field regime, pulsars allow high precision tests that will maintain their importance even in the era of gravitation wave observations with groundbased detectors. This contribution summarise the methods and status of the field and provides a brief outlook into the future.
\end{abstract}

Keywords. gravitation, relativity, (stars:) pulsars: general

\section{Introduction}

Since their discovery in 1967, pulsars have been used as tools for many different applications; one of the most successful one is the usage of pulsars to test theories of gravity. The idea is straightforward: if the pulsar is in orbit with a binary companion, we use the measured variation in the arrival times of the received signal to determine and trace the orbit of the pulsar about the common centre of mass as it moves in the local curved spacetime and in the presence of spin effects. While, strictly speaking, binary pulsars move in the weak gravitational field of a companion, they do provide precision tests of the (quasi-stationary) strong-field regime. This becomes clear when considering that the majority of alternative theories predicts strong self-field effects, which would clearly affect the pulsars' orbital motion. Hence, tracing their fall in a gravitational potential, we can search for tiny deviations from GR, providing us with unique precision strong-field tests of gravity. In fortunate orbital arrangements, we can also use the signal of the pulsar to probe its propagation in the strongly curved spacetime around the companion.

Figure 1 summarises tests today and places them in context with other methods: Pulsar tests go beyond the quasi-stationary weak-field regime of the solar system and test the quasi-stationary strong-field regime, extending also to the radiative regime with those systems where the effects of gravitational wave emission can be studied. The highlyrelativistic regime is currently a domain probed only by the successful LIGO/VIRGO detections. However, with Pulsar Timing Arrays (PTAs) described elsewhere in these proceedings (see contribution by Lee) pulsar observations will also enter this regime.

\section{Method}

Tests can be conducted in a strong-field extension of the "Parameterised PostNewtonian" (PPN) formalism (see Will 2014), probing concepts and principles deeply embedded in theoretical frameworks. Alternatively, as described here, we can perform tests for a given theory and check if it is consistent with the observations. This is done by describing relativistic effects that can be observed in a theory-independent fashion. 


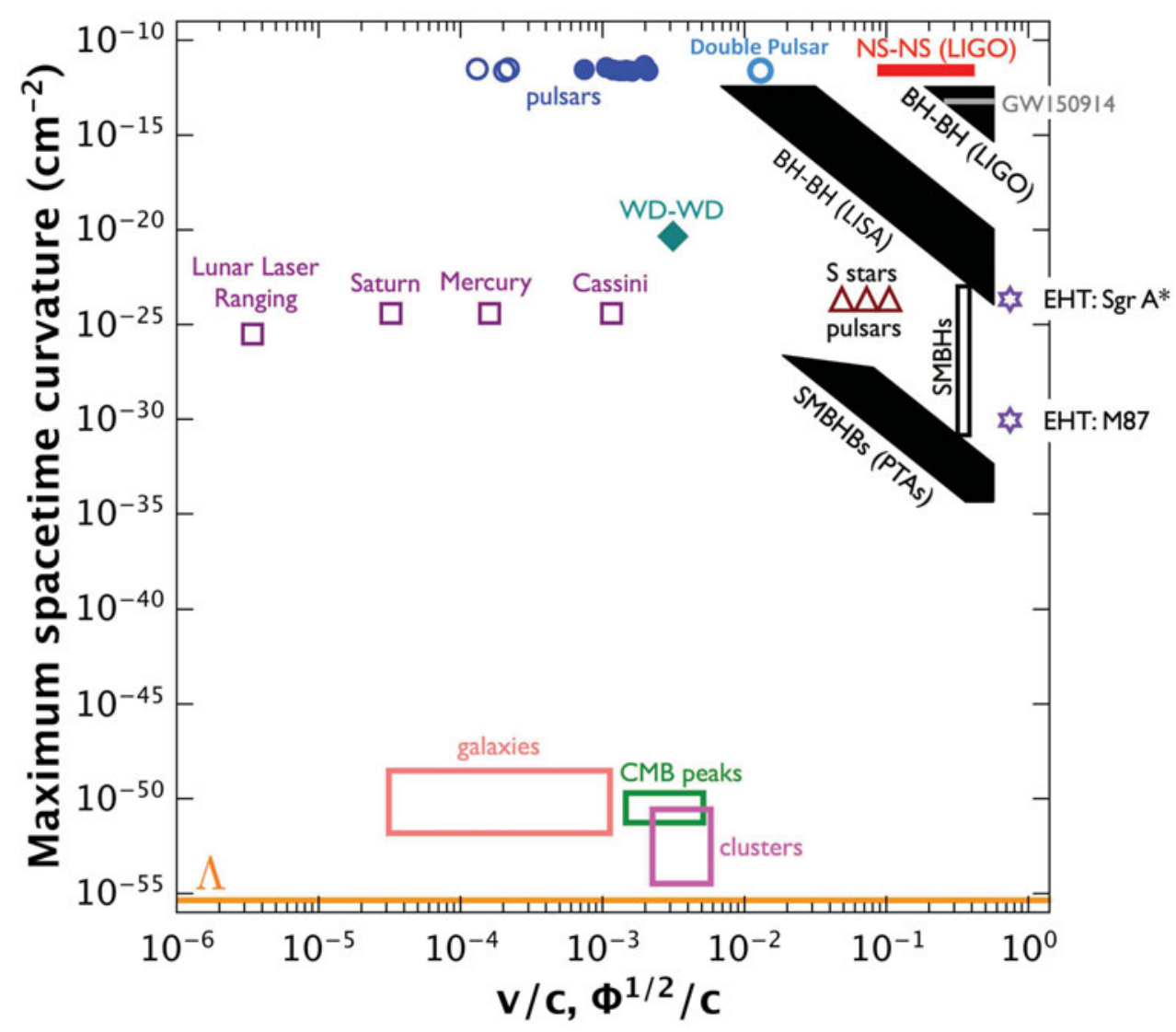

Figure 1. Parameter space of observations and tests of gravity. On the $\mathrm{x}$-axis, $\mathrm{v}$ denotes the typical velocity of the system's components while $\Phi$ denotes the gravitational potential being probed by photons propagating in the corresponding spacetime. On the y-axis we have the maximum spacetime curvature (taken at the horizon for black holes) in the system as a measure of how much the system deviates from flat spacetime. Filled areas indicate gravitational wave tests, while hollow areas stand for quasi-stationary tests, including accretion onto compact objects. The most right hollow blue circle stands for the Shapiro delay test in the Double Pulsar. Figure provided by N. Wex.

This is done by introducing so-called "Post-Keplerian" (PK) parameters that are included in the timing model to accurately describe the measured pulse times-of-arrival (see e.g. Lorimer \& Kramer 2005 for more details). For instance, they describe a periastron advance as a time derivative of the angle of periastron $(\dot{\omega})$ or the change of the orbital period due to a decay of the orbit caused by gravitational wave emission $\left(\dot{P}_{b}\right)$. Other PK parameters describe a combination of a second-order doppler shift with gravitational redshift $(\gamma)$, a Shapiro-delay due to the curvature of space-time around the companion $\left(s\right.$ and $r$ ), or a relativistic deformation of the orbit $\left(\delta_{\theta}\right)$ (Lorimer \& Kramer 2005). A theory is tested when its prediction for the value of a PK parameter can be compared to its measurement from timing observations.

We can expect the theory to describe PK parameters as a function of the measured Keplerian (K) parameters and the two (apriori unknown) masses of the binary components in the system, $m_{p}$ and $m_{c}$. The functional form $P K=f_{P K}\left(m_{p}, m_{c}, K\right)$ will usually differ for a given theory of gravity, but with two PK parameters measured, one can solve for the two mass values. If the theory is a correct description of the data, these 

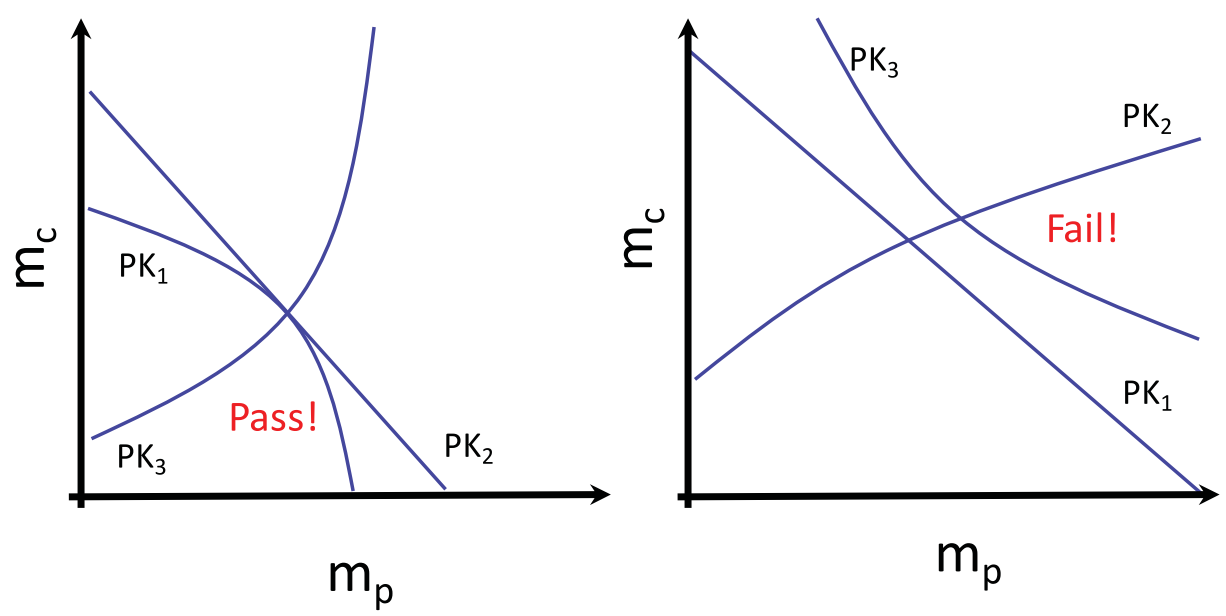

Figure 2. Concept of testing theories of gravity by measuring Post-Keplerian (PK) parameters in a binary system. Each PK parameter describes a line in a "mass-mass diagram" where the companion mass $m_{c}$ is plotted versus the pulsar mass $m_{p}$. The functional dependence for each PK-line depends on the given theory of gravity and the measured Keplerian parameters. If the theory is capable of describing the experiment correctly, all lines should intersect in a single point (left panel), marking the true combination of pulsar and companion mass $\left(m_{p}, m_{c}\right)$. In cases where the PK-lines do not intersect in a single point (right panel), the theory is falsified. In reality, each line has a certain thickness, indicating the measurement uncertainty of the PK parameters (NB: the uncertainty in the measured Keplerian parameters is usually orders of magnitude smaller). As every pair of $N$ measured PK parameters defines an intersection point, a given system allows $(N-2)$ independent tests of theory. Other constraints derived independently or via different methods can also be displayed in the diagram to provide additional constraints. This usually includes a region of the mass-mass diagram excluded by the value of the mass-function and the condition that $\sin i \leqslant 1$, or the measurement of the mass ratio $\left(m_{p} / m_{c}\right)$ like in the Double Pulsar, or an independent measurement of the orbital inclination angle $i$ as such. As each PK line also depends on the Keplerian parameters (i.e. the configuration of the system), each binary system will produce its own unique mass-mass diagram, providing independent and complementary tests of the same theory.

two masses should accurately predict the value for any other PK parameter. Thereby, each additional PK parameter measured has the potential for falsifying the tested theory. A theory, which produces the same pair of mass values from any combination of two PK parameters, is consistent with the data, but should continued to be tested with different systems under different conditions. This way of testing a given theory of gravity can be displayed graphical in a so-called mass-mass diagram, where each of the $N$ measured PK parameters represents a line, showing its dependence on the two masses, i.e. $m_{c}=f_{P K}^{-1}\left(m_{p}, K, P K\right)$. We expect a correct theory to produce lines that all intersect in the same $\left(m_{p}, m_{c}\right)$ point. Since every pair of two PK parameters defines a (potentially different) intersection point, we have $N-2$ independent tests, where in each case the intersection point could differ (see Fig. 2).

\section{Current status}

The first time that the method described above could be applied was the Hulse-Taylor pulsar, B1913+16 Hulse \& Taylor (1975). Up to recently, only three PK parameters were measurable $\left(\dot{\omega}, \gamma, \dot{P}_{b}\right)$, but clearly providing the first evidence for gravitational wave emission (Taylor \& Weisberg 1982). Due to an extended data set and the slow 
precession of the orbit with respect to us as observers, a Shapiro delay can be detected now (Weisberg \& Huang 2016). There are even indications of the first successful detection of the relativistic deformation of the orbit (i.e. a measurement of $\delta_{\theta}$ ). The precision of the test using the initial three PK parameters is still limited by the ability to correct for extrinsic acceleration effects, which alter the observed $\dot{P}_{b}$ value from that solely caused by the effect of gravitational damping (e.g. Lorimer \& Kramer 2005). Improved knowledge of the Galactic gravitational potential provided by astrometric surveys (e.g. by ESA's GAIA mission) promises to increase the precision of this particular radiative test.

Fifty years after the discovery of pulsars, and 44 years after the discovery of the first binary pulsar, gravitational wave damping is routinely observed in appropriate systems. At this meeting, two new exciting systems were announced (see contributions by Cameron and Ferdman), indicating that indeed many more system will be found with more sensitive telescopes. Figure 3 summarises the situation at the beginning of the conference. It is worth noting that the sample is no longer constrained to double neutron star systems, but that quite a few pulsar-white dwarf systems are also among this exclusive group of binary pulsars.

The system with the by far most precise measurement of gravitational wave damping is also the most-over constrained system to perform tests as described above: PSR J0737-3039, also known as the Double Pulsar (Burgay et al. 2003, Lyne et al. 2004), contains to active radio pulsars orbiting each other in 147 minutes. Even though the orbit is less eccentric than that of PSR B1913+16 (resulting in a smaller acceleration around periastron), the orbit is more compact. With a much smaller distance than the HulseTaylor pulsar, extrinsic acceleration effects are also much smaller, so that the current precision of measurement for $\dot{P}_{b}$ is better than $0.1 \%$. This allows for a test of the lowest order of GR's quadrupole formula for gravitational wave emission that is about three orders of magnitude more precise than what gravitational wave detectors can achieve now and in the foreseeable future. In contrast, higher orders of the quadrupole formula are inaccessible in the radiative regime of the Double Pulsar and can only be tested by LIGO/VIRGO currently. The combination of constraints, however, promises to be extremely useful. For a detailed description see the upcoming publication by Kramer et al. (in prep.).

The Double Pulsar has a total of five PK parameters measured $\left(\dot{\omega}, \gamma, \dot{P}_{b}, s, r, \Omega_{\text {geo }}\right)$ with a sixth one $\left(\delta_{\theta}\right)$ emerging as in PSR B1913+16 (see Kramer et al., in prep.). The PK parameter $\Omega_{\text {geo }}$ is the rate of relativistic spin precession for the unrecycled pulsar B in the system. It was measured with a precision of $13 \%$ using the change in the eclipse pattern produced by the rotating magnetosphere of pulsar B, blocking the light of A intermittently during conjunction (Breton et al. 2008). This precession of the spin axis of pulsar B meant that in 2008 the pulsar moved out our line-of-sight (Perera et al. 2010). Depending on the pulsar's beam shape, it will eventually become visible again. Additional new effects related to light propagation in the strongly curved spacetime of pular B have now been detected and will be presented by Kramer et al. (in prep.).

The above method is applicable to all theories of gravity, where the PK parameters can be written in form of the two unknown masses. Measuring the orbital decay due to gravitational wave emission is often a very sensitive tool. Unlike GR, alternative theories, such as those violating the Strong Equivalence Principle (SEP), will, in addition to quadrupolar emission and higher multipoles, also emit dipolar GWs due to an effective dipole from additional gravitational "charges". In double neutron star systems (DNSs), with two (similar) neutron stars in the system, the mass dipole is naturally much smaller than in the case of systems, where the compactness of the binary components is sufficiently different. An ideal case to test for the emission of dipolar GWs would be a 


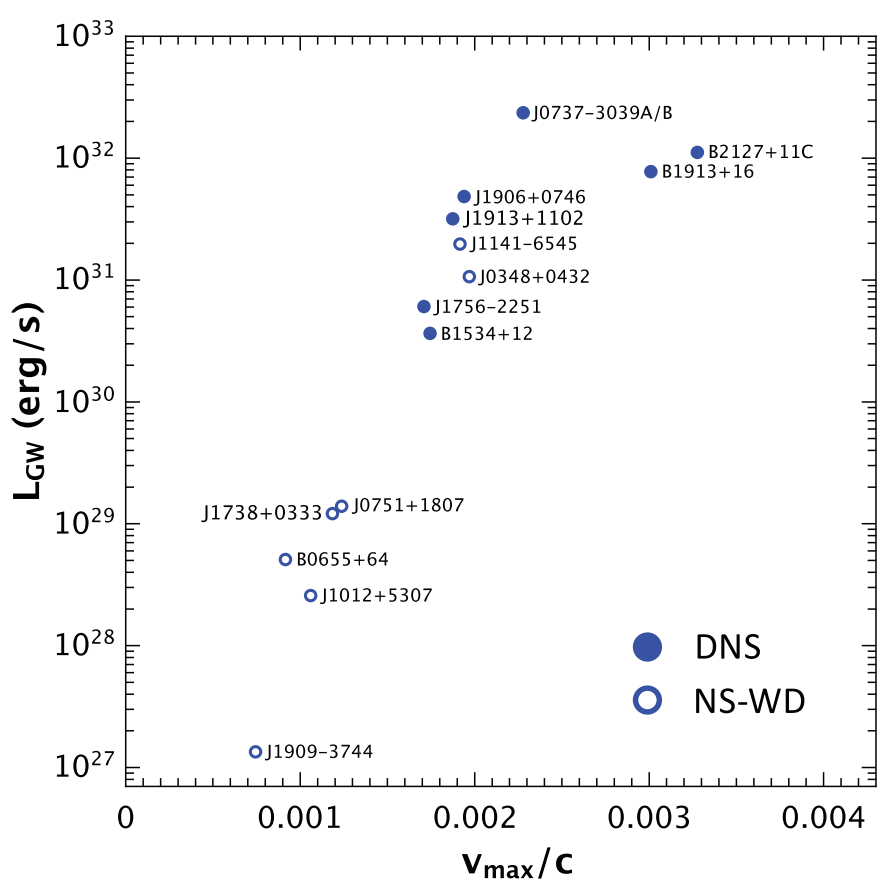

Figure 3. Systems with detected gravitational wave damping known at the time of the conference. See the contributions by Cameron, Ferdman and Lynch for further potential systems. Figure provided by N. Wex.

pulsar-black hole system. Until we find one, one can make good usage of pulsar-white dwarf systems.

Pulsar-white dwarf systems are much more common than DNSs, where the potential progenitor binary system usually gets disrupted in the supernova explosion forming the second-born neutron star. In pulsar-white dwarf system, the period of mass transfer, where matter is accreted on the first-born neutron star, spinning it up to few milliseconds period, is long and often leads to a wide, nearly circular system. In some cases, however, the orbit is compact and also shows relativistic effects like in the case of PSR J0348+0432. The orbital period of this system is only 15 seconds longer than that of the Double Pulsar, but it is also intriguing for another reason. The Helium white dwarf of the system is in orbit around the most massive neutron star known! With a mass of about 2 solar masses, the sheer existence of this neutron star challenges most equation-of-states of super-dense matter. For tests of alternative theories of gravity, on the other hand, the combination of large mass and compact relativistic orbit delivers valuable constraints, e.g. on the coupling strength of a potential gravitational scalar field to matter, or on the existence of dipolar GW radiation. So far, again, the observations are consistent with GR but exclude more and more parameter space for theories other than GR Antoniadis et al. (2013).

Combining such results from further pulsar-white dwarf systems with future measurements of neutron star mergers by LIGO/VIRGO provides constraints on non-perturbative strong-field effects in scalar-tensor gravity (Shao et al. x2017).

\section{Summary \& Outlook}

In order to learn more about methods to tests theories of gravity, I refer to other contributions in these proceedings, especially to contributions by Archibald and collaborators 
on the triple system, and also to reports by Desvignes et al. on relativistic spin precession in PSR J1906+0746.

In terms of precision, binary pulsars will provide the best tests of GR and its alternative for the foreseeable future, especially with FAST, MeerKAT and other telescopes coming online (see contributions by Barr, Li and Keane). Ultimately, the SKA will produce even better systems and even more precision. We can also expect that with the SKA, PTA detection of $\mathrm{nHz}$-gravitational wave signals will be routine, so that at this point, also pulsar observations will enter the highly relativistic regime (see contribution by Lee).

Results obtained from pulsars can be combined successfully with those of other methods. Binary pulsar tests and LIGO/VIRGO observations are perfectly complementary already. Observations of the black hole in the centre of our Galaxy will additionally produce completely new constraints from the "shadow" of the event horizon. Especially if we were to find pulsars orbiting Sgr A*, we would obtain an unprecedented tool to test the properties of black hole spacetime, using the black hole image to test the near-field, and orbiting pulsars to probe the far-field in a combined fashion. See the contribution by Torne et al. for more details.

\section{Acknowledgements}

I am grateful to Norbert Wex for the provision of figures and many useful discussions. I also thank the whole team observing the Double Pulsar over the last decade. Financial support by the European Research Council for the ERC Synergy Grant BlackHoleCam (ERC-2013-SyG, Grant Agreement no. 610058) is gratefully acknowledged.

\section{References}

Antoniadis J. et al., 2013, Science, 340, 448

Breton R. P. et al., 2008, Science, 321, 104

Burgay M. et al., 2003, Nature, 426, 531

Hulse, R. A. \& Taylor, J. H. 1975, ApJ, (Letter) 195, L51

Lorimer, D. R. \& Kramer, M. 2005, Handbook of Pulsar Astronomy, CUP

Lyne A. G. et al., 2004, Science, 303, 1153

Perera B. et al., 2010, ApJ, 721, 1193

Taylor, J. H. \& Weisberg, J. M. 1982, ApJ, 253, 908

Shao, L., et al. 2017 Phys. Rev. X, in press (arXiv:1704.07561)

Weisberg \& Huang, Y. 2016, ApJ, 829, 55

Will, C. M. 2014, Living Reviews in Relativity, 17, 4 